\title{
Disseminated Nocardiosis Initially Manifesting as Visual Field Defects
}

Tamio Okimoto, Yukari Tsubata, Mika Nakao and Takeshi Isobe

Key words: endogenous endophthalmitis, disseminated nocardiosis, malignant mesothelioma

(Intern Med 58: 623-624, 2019)

(DOI: 10.2169/internalmedicine.1601-18)
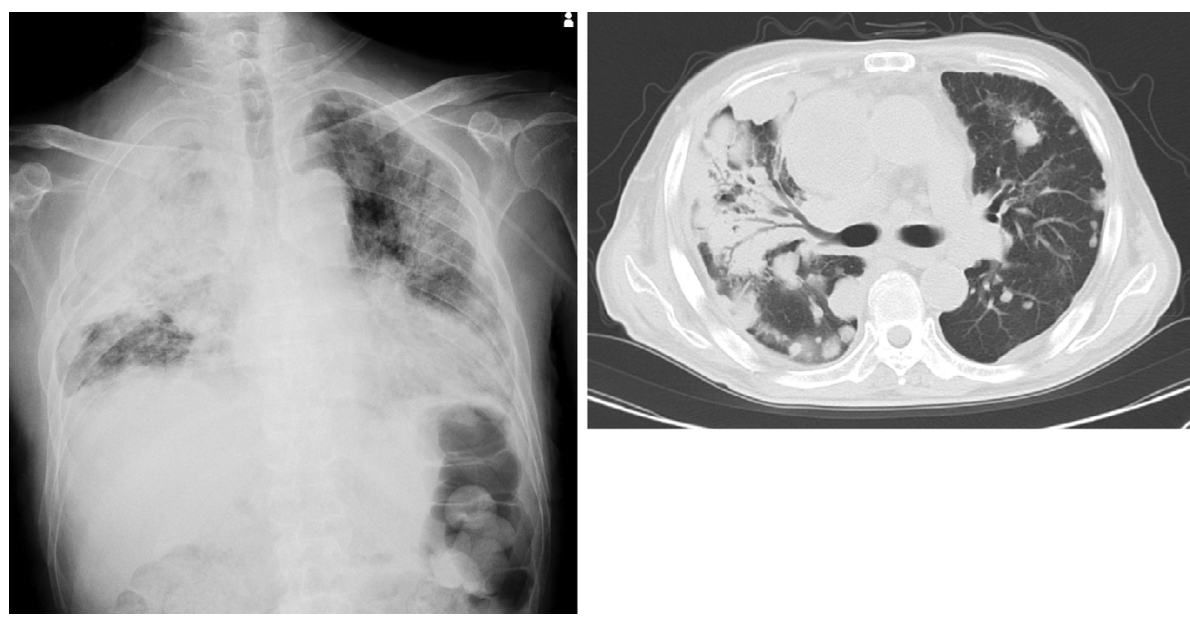

Picture 1.

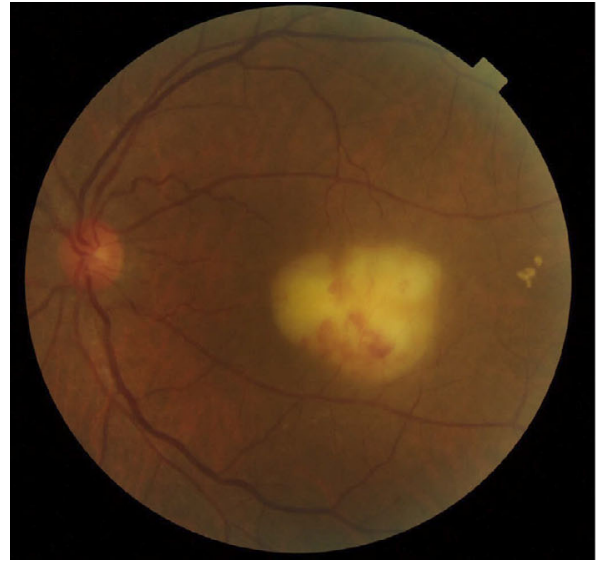

left

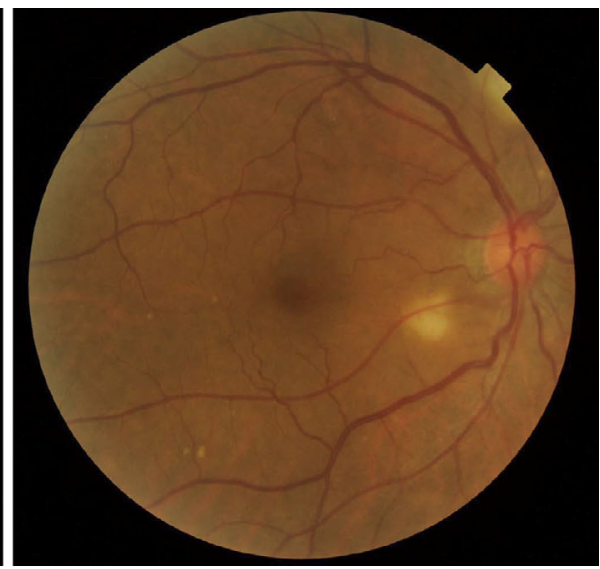

right

Picture 2.

A 70-year-old man with advanced malignant mesothelioma presented with visual field defects and a fever of 3 days duration. He had completed chemotherapy 8 months prior to admission and his tumor was progressive, with enlarging metastatic pulmonary nodules. He was taking betamethasone $(2 \mathrm{mg})$ to maintain his performance status.

Department of Internal Medicine, Division of Medical Oncology and Respiratory Medicine, Shimane University Faculty of Medicine, Japan Received: May 27, 2018; Accepted: July 9, 2018; Advance Publication by J-STAGE: September 12, 2018

Correspondence to Dr. Tamio Okimoto, okimoto@med.shimane-u.ac.jp 
Chest radiography and computed tomography showed new right upper lobe infiltration (Picture 1); sputum and blood cultures were positive for Nocardia cyriacigeorgica. Enhanced head CT and MRI did not show any findings suggestive of brain abscess or metastasis. A fundoscopic examination revealed bilateral exudative plaques (Picture 2). We diagnosed the patient to have disseminated nocardiosis with endogenous endophthalmitis and administered doripenem and sulfamethoxazole-trimethoprim. Although his symptoms improved, the patient subsequently died due to respiratory failure on the 18th day after admission.

The computed tomography findings and symptoms of disseminated nocardiosis are nonspecific (1), rendering detection difficult, especially in patients with thoracic malignan- cies. It is therefore important to note that visual field defects may be an initial manifestation of disseminated nocardiosis.

The authors state that they have no Conflict of Interest (COI).

\section{Reference}

1. Kurahara Y, Tachibana K, Tsuyuguchi K, Akira M, Suzuki K, Hayashi S. Pulmonary nocardiosis: a clinical analysis of 59 cases. Respir Investig 52: 160-166, 2014.

The Internal Medicine is an Open Access journal distributed under the Creative Commons Attribution-NonCommercial-NoDerivatives 4.0 International License. To view the details of this license, please visit (https://creativecommons.org/licenses/ by-nc-nd/4.0/).

(C) 2019 The Japanese Society of Internal Medicine Intern Med 58: 623-624, 2019 